\title{
STATISTICS OF GRAVITATIONAL MICROLENSING MAGNIFICATION. I. TWO-DIMENSIONAL LENS DISTRIBUTION
}

\author{
Lev Kofman \\ Institute for Astronomy, University of Hawaii, 2680 Woodlawn Drive, Honolulu, HI 96822 \\ Nick Kaiser \\ CIAR Cosmology Program, Canadian Institute for Theoretical Astrophysics, University of \\ Toronto, 60 St. George Street, Toronto, Ontario M5S 1A7, Canada \\ Man Hoi Lee \\ Department of Physics, Queen's University, Kingston, Ontario K7L 3N6, Canada \\ and \\ Arif Babul \\ Department of Physics, New York University, 4 Washington Place, New York, NY 10003
}

\begin{abstract}
The propagation of light of distant sources through a distribution of clumpy matter, acting as point mass lenses, produces multiple images that contribute to the total brightness of the observed macroimages. In this paper we refine the theory of gravitational microlensing for a planar distribution of point masses. In the second accompanying paper, we extend the analysis to a three-dimensional lens distribution.

In the two-dimensional case, we derive the probability distribution of macroimage magnification, $P(A)$, at high magnification $\left(A-1 \gg \tau^{2}\right)$ for a low optical depth $(\tau \ll 1)$ lens distribution by modeling the illumination pattern as a superposition of the patterns due to individual "point mass plus weak shear" lenses. A point mass lens perturbed by weak shear $S$ produces an astroid-shaped caustic. We show that the magnification cross-section $\sigma(A \mid S)$ of the point mass plus weak shear lens obeys a simple scaling property and provide a useful analytic approximation for the cross-section. By convolving this cross-section with the probability distribution of the shear due to the neighboring point masses, we obtain a caustic-induced feature in $P(A)$ which also exhibits a simple scaling property. This feature results in a $20 \%$ enhancement in $P(A)$ at $A \approx 2 / \tau$.
\end{abstract}


In the low magnification $(A-1 \ll 1)$ limit, the macroimage consists of a single bright primary image and a large number of faint secondary images formed close to each of the point masses. The magnifications of the primary and the secondary images can be strongly correlated. Taking into account the correlations, we derive $P(A)$ for low magnification and find that $P(A)$ has a peak of amplitude $\sim 1 / \tau^{2}$ at $A-1 \sim \tau^{2}$. The low magnification distribution matches smoothly to the high magnification distribution in the overlapping regimes $A \ll 1 / \tau$ and $A-1 \gg \tau^{2}$.

Finally, after a discussion of the correct normalization for $P(A)$, we combine the high and low magnification results and obtain a practical semi-analytic expression for the macroimage magnification distribution $P(A)$. This semi-analytic distribution is in qualitative agreement with the results of previous numerical simulations, but the latter show stronger caustic-induced features at moderate $A$ for $\tau$ as small as 0.1. We resolve this discrepancy by re-examining the criterion for low optical depth. A simple argument shows that the fraction of caustics of individual lenses that merge with those of their neighbors is approximately $1-\exp (-8 \tau)$. For $\tau=0.1$, the fraction is surprisingly high: $\approx 55 \%$. For the purpose of computing $P(A)$ in the manner we did, low optical depth corresponds to $\tau \ll 1 / 8$.

Subject headings: gravitational lensing

\section{INTRODUCTION}

Gravitational lensing provides a powerful independent tool to probe the distribution of matter in the universe and in individual astronomical objects. There are several situations in which the lensing objects can be modeled as an ensemble of point masses that produce multiple images (microimages) of distant sources. If the angular separations of the microimages are too small to be resolved observationally, one deals with the macroimage, which is the superposition of the microimages. A well-known example of gravitational microlensing is the effect of individual stars in a galaxy on the lensing properties of the galaxy (Young 1981). If the dark matter in the galaxy is in the form of compact objects, the optical depth for microlensing could be even higher. Indeed, the microlensing of stars in nearby galaxies is being used to probe for the possible existence of massive compact halo objects (MACHOs) in our Galaxy (Paczyński 1986b; Alcock et al. 1993; Aubourg et al. 1993). Another interesting possibility is that a substantial fraction of the dark matter in the universe is in the

form of compact objects. Then the universe as a whole has a significant optical depth to 
microlensing (Press \& Gunn 1973). Finally, microlensing can arise in a globular cluster if there are brown dwarfs present (Paczyński 1994).

The main motivation of this study is to give a systematic theory of gravitational microlensing by a random distribution of point masses. There are different regimes of microlensing and they often require different types of modeling. First, one has to distinguish the cases of low $(\tau \ll 1)$, moderate $(\tau \sim 1)$, and high $(\tau \gg 1)$ optical depth, where optical depth $\tau$ is the fraction of the sky covered (locally) by the Einstein circles of all contributing lenses: $\tau=\pi \sum n_{i} x_{E, i}^{2}$, where $n_{i}$ is the projected (onto the observer plane) surface number density of lenses with Einstein radius $x_{E, i}$ (see $\S 2$ ). Second, the spatial distribution of the lenses is usually either "compact" (e.g., stars in a distant galaxy) or "extended" (e.g., cosmologically distributed compact objects). A lens distribution is compact if the lenses are distributed in a region of scale $R$ which is much smaller than the other distances (those between the source and the lenses and between the observer and the lenses) in the problem; it is extended if $R$ is comparable to the other distances in the problem.

In the case of compact lens distributions, the deflection of light rays is generally small and essentially occurs in the vicinity of the lens distributions. Consequently, a compact lens distribution is usually approximated as a planar (two-dimensional) structure corresponding to its projection onto a lens plane that is oriented perpendicular to the line of sight between the observer and the source. This "single lens plane" approximation allows us to pose and explore well-defined questions concerning the caustic structure of the lens configuration, the resulting illumination pattern, the probability distribution of macroimage magnification, the temporal variation of the observed brightness of a source (which is the magnification profile along a track through the illumination pattern), etc. And while these issues have been explored in great detail both analytically and via computer simulations (Kaiser 1992; Mao 1992; Rauch et al. 1992; see also the book by Schneider, Ehlers, \& Falco 1992 and references therein), by no means have all the outstanding issues been resolved.

The greatest progress has been made in understanding the properties of single plane lensing under low optical depth conditions. One of the earliest approaches (e.g., Turner, Ostriker, \& Gott 1984) advocated modeling the low- $\tau$ illumination pattern as a superposition of what one would obtain for a collection of point masses acting independently. An isolated point mass lens produces a circularly symmetric illumination pattern with a divergent spike at the origin. Its differential cross-section for magnification $A, \sigma_{0}(A)$, is proportional to the mass of the lens and scales as $A^{-3}$ for $A \gg 1$. A simple superposition of the differential cross-sections implies that the probability distribution of macroimage magnification $P(A)$ is (Paczyński 1986b)

$$
P(A) d A=2 \tau A^{-3} d A \quad \text { for } A \gg 1
$$


or more generally,

$$
P(A) d A=2 \tau\left(A^{2}-1\right)^{-3 / 2} d A \quad \text { for } A-1 \gg \tau^{2}
$$

It is well known, however, that neither the $\tau$ - nor the $A$-dependence is correct in the above distributions, even in the limit of high magnification. As shown by Nityananda \& Ostriker (1984; also Chang \& Refsdal 1984), each lens is subject to an external shear from the macroscopic mass distribution as well as its nearest neighbors. An isolated point mass lens produces a degenerate point-like caustic. External shear breaks the degeneracy and as we shall demonstrate in this paper, the resulting caustic has the shape of an "astroid" and its width is proportional to the dimensionless magnitude $S$ of the shear perturbation. The corresponding magnification cross-section $\sigma(A \mid S)$ shows a strong feature at $A \sim 1 / S$. This suggests that there ought to be a feature in $P(A)$ at $A \sim 1 / \tau$ since it can readily be shown that both the typical magnitude of the macroscopic shear and its random component are of order $\tau$. The natural extension of the superposition approach, therefore, is to model the illumination pattern as a superposition of the patterns due to individual "point mass plus weak shear" lenses. Briefly, the resulting probability distribution of magnification is a convolution of the magnification cross-section $\sigma(A \mid S)$ for a single lens with the probability distribution function for the shear $p(S)$. In a subsequent section, we pursue this approach and discuss the properties of the $P(A)$ thus acquired.

Schneider (1987a) adopted a very different approach in attempting to determine the asymptotic behavior of $P(A)$ in the limit of high magnification. He argued that the high magnification events are dominated by observers lying close to the fold caustics and hence, the illumination pattern is dominated by two images of nearly equal brightness. This greatly simplifies the problem. Instead of having to identify all the microimages that make up a macroimage and then calculate the probability distribution function for their total magnification, one can simply use the probability distribution function for the individual image magnification. The latter can be calculated regardless of the optical depth. This approach, however, has one drawback and that is the difficulty in quantifying how large $A$ must be before $P(A)$ is well approximated by its asymptotic form. In the low optical depth derivation discussed above, the asymptotic form is a good approximation for $A \gg \tau^{-1}$. Nonetheless, it should be noted that Schneider's derivation is the only rigorous analytic result for finite $\tau$.

Understanding the behavior of $P(A)$ at low magnification (i.e., at $\delta A \equiv A-1 \sim \tau^{2}$ ) is also problematic. The distribution quoted above (eq.[2]) is not properly normalized as the integral $\int d A P(A)$ diverges as the lower limit of the integral approaches $A=1$. One rather cavalier approach is simply to impose a cut-off at some $A_{\min }$ such that the total probability is unity. Schneider (1987b) adopted a more sophisticated approach. He argued that a typical observer (i.e., one who is not particularly well aligned with any single lens) will see one 
primary image close to the unperturbed position of the source and a large number of faint secondary images (one close to each lens) produced by strongly deflected rays - the so-called "diffuse" component. Then the total macroimage magnification associated with the lensing event is $A=A_{0}+\sum_{i=1}^{N} A_{i}$, where $A_{0}=1+\delta A_{0}$ is the magnification of the primary image and $A_{i}(i>0)$ is the magnification of the secondary images. Assuming that $\delta A_{0}$ and $\sum_{i=1}^{N} A_{i}$ are statistically independent, Schneider obtained a $P(\delta A)$ that exhibits a peak at $\delta A \sim \tau^{2}$, is correctly normalized, and has the same $A$-dependence as equation (2) for $A-1 \ll 1$. Unfortunately, the two expressions differ in amplitude, a discrepancy that arises because the magnifications of the primary and secondary images are not independent. In fact, we will show rigorously that the magnifications can be strongly correlated.

In the above paragraphs, we have hinted at the various calculations and discussion that are to be presented in this paper. Our overall goal is to find an expression for $P(A)$ in the low optical depth limit that represents a synthesis of the ideas discussed above and that is both useful as well as physically motivated. Our approach is a two-pronged one. For $\delta A \gg \tau^{2}$, we perform the superposition of the point mass plus weak shear lenses, allowing for the random mutual shear perturbation. In $\S \S 3.1-2$ we calculate the cross-section of an individual point mass plus weak shear lens which shows strong caustic-induced features. In $\S 3.3$ we consider the random superposition of the point mass plus weak shear lenses. We find that the random shear tends to smear out the strong caustic-induced features of the individual lenses and that the deviations from equation (2) around $A \sim 1 / \tau$ are at the $20 \%$ level. We also find a simple scaling property for the caustic-induced "bump" for low optical depth. For low magnification, we resolve the discrepancy in Schneider's (1987b) calculation by allowing for the correlations between the primary and secondary images $(\S 4)$. This gives a formula which is valid for $A-1 \ll 1$ and which agrees with the expression for $\delta A \gg \tau^{2}$ in both slope and amplitude. An expression for $P(A)$ that includes all the relevant effects is given in $\S 5.1$. We compare this semi-analytic $P(A)$ with the numerical results of Rauch et al. (1992) in $\S 5.2$. While the caustic-induced bump in the semi-analytic $P(A)$ agrees qualitatively with the bump found in the numerical simulations, the numerical results show stronger caustic-induced features at moderate $A$ for $\tau$ as small as 0.1 . The additional features are due to caustic configurations more complicated than the simple astroids of the point mass plus weak shear lenses. To understand the contribution from the more complicated caustic configurations at small $\tau$, we consider the leading additional contribution from close pairs of point masses with merged caustics. In $\S 5.3$ we calculate the fraction of point masses whose caustics are not isolated astroids as a function of $\tau$. This gives a simple criterion for the optical depth $\tau$ below which $P(A)$ can be constructed by the superposition of the cross sections of individual lenses.

In the second accompanying paper (Lee et al. 1996; hereafter Paper II), we conduct a 
similar study for the more complicated situation of a three-dimensional distribution of point mass lenses.

\section{LENS EQUATIONS}

We begin by outlining the basic concepts of gravitational lensing, discussing the relevant equations and defining our notation. For the latter, we have adopted the notation of Kaiser (1992), some of which differs slightly from those in general use.

For simplicity, we shall only consider gravitational lensing in an Einstein-de Sitter cosmological background weakly perturbed by the gravitational field of the lenses. The line element for such a universe is

$$
d s^{2}=a^{2}(\eta)\left[(1+2 \phi) d \eta^{2}-(1-2 \phi)\left(d \chi^{2}+\chi^{2} d \Omega^{2}\right)\right] .
$$

Let the conformal time $\eta=1$ at the present. The scale factor is $a=a_{0} \eta^{2}$, and the Hubble parameter is $H=H_{0} \eta^{-3}$ with $H_{0}=2 / a_{0}$. In this convention, the unit comoving length is $a_{0}=6000 h^{-1} \mathrm{Mpc}$, where $h \equiv H_{0} /\left(100 \mathrm{~km} \mathrm{~s}^{-1} \mathrm{Mpc}^{-1}\right)$. The Newtonian (peculiar) potential $\phi$ induced by the density inhomogeneities is given by Poisson's equation (in comoving coordinates):

$$
\nabla^{2} \phi(\chi)=6 \eta^{-2} \Delta(\chi), \quad \Delta(\chi) \equiv(\rho(\chi)-\bar{\rho}) / \bar{\rho}
$$

Let us consider an isotropic point source at the origin of our coordinates and an observer plane which is perpendicular to the $z$-axis and at a comoving distance $\chi_{\text {so }}$ from the source (see Fig. 1). In the absence of perturbations, the observer plane would be uniformly illuminated and a ray which leaves the source with angle $\boldsymbol{\theta}=\left(\theta_{1}, \theta_{2}\right)$ would pierce the observer plane at $\boldsymbol{x}=\chi_{s o} \boldsymbol{\theta}$. This defines our planar Lagrangian coordinates $\boldsymbol{x}$. In the presence of density inhomogeneities, the light ray would suffer deflections and the comoving displacement vector $\boldsymbol{s}(\boldsymbol{x}, \chi)$ at distance $\chi$ from the source is given by

$$
\boldsymbol{s}(\boldsymbol{x}, \chi)=-2 \int_{0}^{\chi} d \chi^{\prime}\left(\chi-\chi^{\prime}\right) \boldsymbol{\nabla} \phi\left[\boldsymbol{x}^{\prime}+\boldsymbol{s}\left(\boldsymbol{x}, \chi^{\prime}\right), \chi^{\prime}\right]
$$

where $\boldsymbol{x}^{\prime}=\chi^{\prime} \boldsymbol{\theta}$. For $\chi=\chi_{\text {so }}$, equation (5) defines the mapping from Lagrangian to Eulerian coordinates in the observer plane:

$$
\boldsymbol{r}(\boldsymbol{x})=\boldsymbol{x}+\boldsymbol{s}\left(\boldsymbol{x}, \chi_{s o}\right)
$$

For most compact lens distributions of interest, the deflection of a light ray is small and occurs essentially in the vicinity of the lens. In effect, the deflection can be thought of as 
occurring at a plane that is situated close to the location of the lens(es). In this (single) thin-screen approximation, the lens mapping (6) is a gradient mapping with the displacement vector $\boldsymbol{s}=\boldsymbol{\nabla} \Psi$, where $\Psi$ is an effective surface gravitational potential. For the particular case of single plane lensing by point masses (for simplicity, we assume that all the point masses have the same mass $m$ ),

$$
\boldsymbol{s}(\boldsymbol{x})=-4 G m\left(\frac{\chi_{L o} \chi_{s o}}{a_{L} \chi_{s L}}\right) \sum_{k} \frac{\boldsymbol{x}-\boldsymbol{x}_{k}}{\left|\boldsymbol{x}-\boldsymbol{x}_{k}\right|^{2}}=-x_{E}^{2} \sum_{k} \frac{\boldsymbol{x}-\boldsymbol{x}_{k}}{\left|\boldsymbol{x}-\boldsymbol{x}_{k}\right|^{2}},
$$

where $\boldsymbol{x}_{k}$ is the position of the $k$ th lens projected onto the observer plane (i.e., $\boldsymbol{x}_{k}=\chi_{s o} \boldsymbol{\theta}_{k}$ where $\boldsymbol{\theta}_{k}$ is the angular position of lens $k$ from the source), $a_{L}$ is the scale factor at the redshift of the lens plane, and $\chi_{L o}, \chi_{s o}$, and $\chi_{s L}$ are the comoving distances between the lens plane and the observer plane, between the source and the observer plane, and between the source and the lens plane, respectively (see Fig. 1). In addition,

$$
x_{E} \equiv\left(\frac{4 G m \chi_{L o} \chi_{s o}}{a_{L} \chi_{s L}}\right)^{1 / 2}
$$

is the (Lagrangian) Einstein radius of a point mass on the lens plane. For an ensemble of randomly distributed point masses, the optical depth to microlensing is $\tau=\pi n x_{E}^{2}$, where $n=\left(a_{L} \chi_{s L} / \chi_{s o}\right)^{2} \Sigma / m$ is the projected (onto the observer plane) surface number density of point masses and $\Sigma$ the physical surface mass density of the lenses.

The generic behavior and the properties of gradient mappings such as that defined by equation (6) are relatively well-known. In fact, the gradient mapping has been used to describe a variety of phenomena. The mapping ought to be familiar to the aficionados of the Zel'dovich approximation (Zel'dovich 1970) for describing the growth of cosmological velocities and density inhomogeneities due to gravitational instability. The evolution of the density probability distribution function in the Zel'dovich approximation before significant orbit crossing was studied by Kofman et al. (1994). The two-dimensional mapping has been used to describe the brightness distribution behind a phase screen (similar to the illumination pattern that appears at the bottom of a swimming pool) (Longuet-Higgins 1960; Gurbatov, Malakhov, \& Saichev 1991). In all cases, however, the results give the brightness distribution (or the density of particles) for a single stream. We are not aware of any multiple streaming solution for the brightness distribution at the bottom of a swimming pool, for instance. The situation in microlensing is quite different in that one must solve the gradient mapping equation to locate all the microimages, and then sum the image magnifications to obtain the macroimage magnification. This is much more difficult.

In general, gravitational lensing maps a number of (micro)images with Lagrangian coordinates $\boldsymbol{x}^{1}, \ldots, \boldsymbol{x}^{n}$ onto the same Eulerian coordinate $\boldsymbol{r}$. The observed flux of the image 
with position $\boldsymbol{x}=\boldsymbol{x}^{l}$ is magnified (or amplified) by $A_{l}=1 /\left|\widehat{\mathcal{D}}\left(\boldsymbol{x}^{l}\right)\right|$, where

$$
\widehat{\mathcal{D}} \equiv \frac{\partial \boldsymbol{r}}{\partial \boldsymbol{x}}=\widehat{\mathcal{I}}+\frac{\partial \boldsymbol{s}}{\partial \boldsymbol{x}}
$$

is the deformation tensor (or Jacobian matrix) associated with the lens mapping and $\widehat{\mathcal{I}}$ is the identity matrix. If the individual microimages are not resolved observationally, one deals with the macroimage which has a total magnification (or amplification) $A=\sum_{l=1}^{n} A_{l}$. In Lagrangian space $(\boldsymbol{x})$, the loci of points on which $|\widehat{\mathcal{D}}|=0$ (i.e., infinite magnification) trace out curves that are called critical curves, while the mapping of these curves to Eulerian space $(\boldsymbol{r})$ defines the caustics. In our notation, because we consider a single source and an ensemble of observers, the caustics lie on the observer plane. This is different from the usual practice of considering a single observer and an ensemble of sources, which has caustics on the source plane.

For a given lens configuration, one can define a differential cross-section $\sigma(A)$ such that $\sigma(A) d A$ is the area in the observer plane where the total magnification is between $A$ and $A+d A$. As we shall see, it is also useful to define a "normalized" differential cross-section $\varphi(A) \equiv \sigma(A) / \sigma_{0}(A)$, where $\sigma_{0}(A)$ is the differential cross-section associated with the simple lens configuration of a single isolated point mass (see eq. 111 below). The function $\varphi(A)$ is a measure of the deviations of the cross-section of a more complex lens configuration from that of the single isolated point mass lens. Finally, for lensing by an ensemble of randomly distributed lenses, the generalization of the cross-section is the probability distribution of macroimage magnification $P(A)$.

\section{CAUSTIC-INDUCED FEATURE AT HIGH MAGNIFICATION}

In $\S 3.1$ we solve the lens equation for a point mass lens perturbed by weak shear. This allows us to study analytically the caustic-induced features in the differential cross-section

( $\S 3.2$ ). Finally, in $\S 3.3$, we consider the superposition of the point mass plus weak shear lenses and derive the caustic-induced feature in $P(A)$ at high magnification.

\section{1. "Point Mass Plus Weak Shear" Lens: Solution of the Lens Equation}

The lens equation for an isolated point mass lens located at comoving distance $\chi_{L o}$ from the observer and at the origin of the lens plane is

$$
\boldsymbol{r}=\boldsymbol{x}-x_{E}^{2} \frac{\boldsymbol{x}}{|\boldsymbol{x}|^{2}}
$$


The corresponding differential cross-section is

$$
\sigma_{0}(A)=\frac{2 \pi x_{E}^{2}}{\left(A^{2}-1\right)^{3 / 2}} .
$$

As is well known, the caustic associated with this lens is a degenerate point located at $\boldsymbol{r}=0$, the degeneracy being due to the spherical symmetry of the lens.

The degeneracy is lifted if the point mass is not strictly isolated and is perturbed by shear, as in the typical lensing configuration in single plane low optical depth microlensing. Under low optical depth conditions, lensing (other than low magnification events) is dominated by the point mass closest to the location where the light ray pierces the lens plane and the contribution of the other distant point masses can be treated as perturbations. If we choose a coordinate system such that the dominant lens is located at the origin of the lens plane and expand the perturbations to the first order in $\boldsymbol{x}$, the perturbations correspond to a constant deflection and a small constant shear and the lens mapping (eqs. [6] and [7]) simplifies to

$$
\boldsymbol{r}=\boldsymbol{x}+\boldsymbol{s}(\boldsymbol{x}), \quad \boldsymbol{s}(\boldsymbol{x}) \approx \boldsymbol{d}_{L}(\boldsymbol{x})+\boldsymbol{\alpha}_{L}+\widehat{\mathcal{S}}_{L} \boldsymbol{x}
$$

where

$$
\boldsymbol{d}_{L}(\boldsymbol{x})=-x_{E}^{2} \frac{\boldsymbol{x}}{|\boldsymbol{x}|^{2}}
$$

denotes the influence of the dominant lens, while $\boldsymbol{\alpha}_{L}$ (the constant deflection) and $\widehat{\mathcal{S}}_{L}$ (the shear matrix) represent the perturbative influence of the other lenses on the plane:

$$
\begin{aligned}
\boldsymbol{\alpha}_{L} & =x_{E}^{2} \sum_{k} \frac{\boldsymbol{x}_{k}}{\left|\boldsymbol{x}_{k}\right|^{2}} \\
\widehat{\mathcal{S}}_{L} & =x_{E}^{2} \sum_{k} \frac{1}{\left|\boldsymbol{x}_{k}\right|^{4}}\left(\begin{array}{cc}
x_{k}^{2}-y_{k}^{2} & 2 x_{k} y_{k} \\
2 x_{k} y_{k} & y_{k}^{2}-x_{k}^{2}
\end{array}\right) \equiv S \widehat{\mathcal{T}}\left(\phi_{L}\right) .
\end{aligned}
$$

In the last equation, $S$ is the magnitude of the shear perturbation and

$$
\widehat{\mathcal{T}}\left(\phi_{L}\right)=\left(\begin{array}{rr}
\cos 2 \phi_{L} & \sin 2 \phi_{L} \\
\sin 2 \phi_{L} & -\cos 2 \phi_{L}
\end{array}\right) .
$$

Finally, transforming the Eulerian variable according to $\boldsymbol{r} \rightarrow \boldsymbol{r}^{\prime}=\boldsymbol{r}-\boldsymbol{\alpha}_{L}$ and rotating the coordinate axes such that they coincide with the principal shear axes, i.e., $\phi_{L}=0$, we can cast equation (12) into a more conventional form:

$$
\boldsymbol{r}^{\prime}=\boldsymbol{x}-x_{E}^{2} \frac{\boldsymbol{x}}{|\boldsymbol{x}|^{2}}+S \widehat{\mathcal{T}}(0) \boldsymbol{x}
$$


where

$$
\widehat{\mathcal{T}}(0)=\left(\begin{array}{rr}
1 & 0 \\
0 & -1
\end{array}\right) .
$$

Equation (15) yields a fourth order equation for the image positions (see Appendix A), which has four solutions inside the caustic, and two solutions outside.

For arbitrary value of $S$, it is difficult to proceed any further, especially with regards to determining the cross-section $\sigma(A)$ analytically. Fortunately, the magnitude of shear is typically $S \sim \tau$ and in the limit of low optical depth, is small (see $\S 3.3$ ). In Figure 2 we show the caustic and the contours of constant magnification on the observer plane for a point mass perturbed by weak shear $(S \ll 1)$. (The results shown in Figure 2 were obtained numerically for $S=0.02$.) For $S \ll 1$, one can observe that the entire four-stream region (i.e., the region inside the caustic) has high magnification. The minimum (though high) magnification in this region occurs at $\boldsymbol{r}^{\prime}=0$ and it can be found from equations (A1)-(A3) that

$$
A=\left(S-S^{3}\right)^{-1} \approx S^{-1} \gg 1
$$

at $\boldsymbol{r}^{\prime}=0$ (also Mao 1992). Elsewhere within the caustic, $A$ is even higher and diverges to infinity at the caustic. This means that the light rays that delineate the four-stream region pass very close to the critical curve in Lagrangian space. To first order in $S$, the equation for the critical line (eq.[A4]) can be expressed as

$$
x^{2}=x_{E}^{2}(1+S \cos 2 \vartheta)
$$

where we have used the polar representation $(x, \vartheta)$ for the Lagrangian coordinate $\boldsymbol{x}$. Hence, the critical line is a slightly flattened ellipse. We can study the behavior of the rays that pass near the critical line by considering rays with $\left(x / x_{E}\right)^{2}=1+t$ and $t \ll 1$. If we now rescale the variables: $t^{\prime}=t / S$ and $\varrho^{\prime}=\varrho / S=r^{\prime} /\left(x_{E} S\right)$, equation (A1) reduces at the lowest order in $S$ to a simple quartic equation for $t^{\prime}$ :

$$
t^{\prime 4}-\left(2+\varrho^{\prime 2}\right) t^{\prime 2}+2 \varrho^{\prime 2} t^{\prime} \cos 2 \phi+\left(1-\varrho^{\prime 2}\right)=0 .
$$

In general, equation (19) has either two or four real solutions (corresponding to the positions of the two images outside the caustic or the four images inside). Once the images are identified, their magnifications can be summed to get the macroimage magnification factor:

$$
A\left(\boldsymbol{r}^{\prime}\right)=\frac{1}{2 S} \sum\left|t^{\prime}-\frac{\left(t^{\prime 2}+1\right) \cos 2 \phi-2 t^{\prime}}{t^{\prime 2}-2 t^{\prime} \cos 2 \phi+1}\right|^{-1} .
$$

Therefore, the magnification inside and around the caustic depends on the parameter $S$ in the form $A \propto 1 / S$. This means that the distribution of $A$ over the observers is a function of the combination $S A$ only and any function of $A$ obeys a simple scaling property. 
We can obtain a parametric expression for the caustic in Eulerian space by solving equations (19) and (20):

$$
\begin{aligned}
\varrho^{\prime 2}(\phi) & =\frac{\left(t^{\prime 2}-1\right)^{2}}{t^{\prime 2}-2 t^{\prime} \cos 2 \phi+1}, \\
t^{\prime}(\phi) & =\cos 2 \phi-\left[\sin ^{2} 2 \phi(1+\cos 2 \phi)\right]^{1 / 3}+\left[\sin ^{2} 2 \phi(1-\cos 2 \phi)\right]^{1 / 3} .
\end{aligned}
$$

After some tedious algebra, equation (21) can be simplified to

$$
\varrho^{2 / 3}\left(\cos ^{2 / 3} \phi+\sin ^{2 / 3} \phi\right)=2^{2 / 3} \text {. }
$$

Thus the caustic has the shape of an astroid, with the four cusp catastrophes at $\phi=(n-$ 1) $\pi / 2(n=1, \ldots, 4)$ and at a distance $\varrho^{\prime}=2\left(\right.$ or $\left.r^{\prime}=2 x_{E} S\right)$ from the center of the astroid connected to each other by four fold catastrophes. The caustic shown in Figure 2, which was obtained numerically for $S=0.02$, is described by equation (22).

\subsection{Analytic Determination of the Caustic-Induced Features in $\sigma(A \mid S)$}

One consequence of the scaling relationship noted above is that the caustic-induced features in the differential cross-section of a point mass plus weak shear lens scale as

$$
\sigma(A \mid S)=\sigma_{0}(A) \varphi(S A)
$$

where $\sigma_{0}(A)$ is the differential cross-section of an isolated point mass (eq. 11]) and $\varphi(S A)$ is a function describing the scaling behavior. In Figure 3 we show the "normalized" differential cross-section $\varphi(S A)$ obtained numerically for $S=0.02$ (solid line). The modification of the cross-section at $S A \sim 1$ is quite pronounced (a factor of a few higher or lower) and the deviations persist over a decade in $S A$. These features were first found numerically by Nityananda \& Ostriker (1984; see also Mao 1992). In this subsection, we investigate the properties of $\varphi(S A)$ and find analytically the critical values of $A$ and $\varphi(S A)$. We will also provide an analytic fit to $\varphi(S A)$.

First it can be seen that $\varphi(S A)$ tends towards unity asymptotically at high and low magnifications. As we have noted previously, $\sigma(A \mid S) d A$ is the area in the Eulerian plane between the $A$ and $A+d A$ contours. Essentially, this is equal to the length of the iso-magnification contour corresponding to magnification $A$ multiplied by the incremental separation $d r^{\prime}$ between the $A$ and $A+d A$ contours. It is well known that in the vicinity of the fold caustic (i.e., in the limit of high magnification $A \gg 1 / S), A \propto 1 / \sqrt{r_{c}^{\prime}(\phi)-r^{\prime}}$, where $r_{c}^{\prime}(\phi)$ is the 
distance to the caustic from the center of the astroid. On the other hand, the lengths of the iso-magnification contours are nearly independent of magnification because both in size and in shape the contours asymptotically approach the astroid-shaped caustic. Consequently, $\sigma(A \mid S) d A \propto d r^{\prime} \propto d A / A^{3}$. Since the differential cross-section of an isolated point mass also depends on $A$ according to $\sigma_{0}(A) \propto 1 / A^{3}$ in the high magnification limit, $\varphi(S A)$ tends to a constant value. In fact, to first order in $S$, the magnification cross-sections of the isolated point mass and the point mass plus weak shear cases coincide at high magnification and $\varphi \rightarrow 1$.

At large distances from the astroid, we can solve equations (A1) and (A2) by only considering terms to first order in $S$ and using a perturbation series with respect to $1 / r^{\prime}$. We find that the resulting iso-magnification contours are given by $A \approx 1+2\left(x_{E} / r^{\prime}\right)^{4}+$ $2 S\left(x_{E} / r^{\prime}\right)^{2} \cos 2 \phi$. In the limit of low magnification $A \ll 1 / S$ (but with $A-1 \gg S^{2}$ ), the contours are similar to those associated with an isolated point mass lens. Consequently, the magnification cross-section converges to that of an isolated point mass and $\varphi \rightarrow 1$.

The interesting features in $\varphi(S A)$ occur at $S A \sim 1$. We can understand these features by studying the geometry of the iso-magnification contours on the observer plane (see Fig. 2). As we noted above, the iso-magnification contours at large distances from the astroid are slightly deformed circles. In going from low to high magnification, the quadrupole moment of the contours increases and the iso-magnification contours are continuously deformed. At the first critical value $A_{1}$, the contour osculates the astroid and due to symmetry, touches it at four Eulerian points with coordinates $r^{\prime}=S x_{E}, \phi=(2 n-1) \pi / 4$, and $n=1, \ldots, 4$. There are four images associated with each of these points, and we can determine their Lagrangian coordinates as well as their magnifications from equations (19) and (20). Two of these images have infinite magnification and the other two have finite magnification; it is the latter two that are of interest. From equation (20), we find that the total magnification associated with the images of interest is

$$
A_{1}=2 / 3 \sqrt{3} S .
$$

The next contours of $A$ slightly larger than $A_{1}$ consist of four symmetric arcs around and outside the four cusps and because of the loss of the area inside the astroid the corresponding $\sigma(A) d A$ decreases rapidly. This explains the first break in $\varphi(S A)$ at $S A_{1}$.

Next, we consider the iso-magnification contours inside the caustic, i.e., in the fourimage region. The minimum magnification $A_{2}$ inside the caustic is located at $r^{\prime}=0$, the origin of the Eulerian plane. Using equations (19) and (20), we find that

$$
A_{2}=1 / S \text {. }
$$


[As we mentioned earlier, the exact solution is $A_{2}=1 / S\left(1-S^{2}\right)$ for $0<S<1$, which reduces to $1 / S$ for $S \ll 1$; eq.(17)]. We can also find the magnification in the vicinity of the origin by evaluating equations (19) and (20) for $r^{\prime} \ll S x_{E}$ or $\varrho^{\prime} \ll 1$. We find that the Eulerian position $\left(r^{\prime}, \phi\right)$ is associated with four images with $t_{1,2}^{\prime}=1 \pm\left(r^{\prime} / S x_{E}\right) \sin \phi$ and $t_{3,4}^{\prime}=-1 \pm\left(r^{\prime} / S x_{E}\right) \cos \phi$ and that the total magnification is

$$
A\left(r^{\prime}\right)=\frac{1}{S}+\frac{1}{4 S}\left(\frac{r^{\prime}}{S x_{E}}\right)^{2} .
$$

Therefore, the differential cross-section is

$$
\sigma(A \mid S)=2 \pi r^{\prime} \frac{d r^{\prime}}{d A}=4 \pi x_{E}^{2} S^{3} \Theta(S A-1)+\delta \sigma(A \mid S),
$$

where $\Theta$ is the step function and the term $\delta \sigma(A \mid S) \sim A^{-7 / 2}$ (Mao 1992) takes into account the contribution to the differential cross-section from contours with $A>A_{2}$ that are outside the caustic and in the vicinity of the cusps (see Fig. 2). The discontinuous jump at $A_{2}$ described by equation (27) is in good agreement with the jump of height 2 in $\varphi(S A)$ shown in Figure 3.

Finally, we provide a simple analytic fit (with three pieces for the different $A$ intervals) to the numerically obtained differential cross-section of the point mass plus weak shear lens shown in Figure 3:

$$
\begin{aligned}
\varphi(S A) & =1+7.7(S A)^{3.5} & & \text { for } A \leq A_{1}, \\
& =\frac{0.17}{(S A-0.33)^{1 / 2}}+\frac{0.023}{(S A-0.33)} & & \text { for } A_{1} \leq A \leq A_{2}, \\
& =1+\frac{0.85}{S A}+\frac{0.37}{(S A)^{5}} & & \text { for } A \geq A_{2} .
\end{aligned}
$$

This fit is shown by the dotted lines in Figure 3.

\subsection{Superposition of Cross-Sections with Distributed Shear}

Let us now consider a collection of point masses (all of the same mass $m$ ) randomly distributed on a single lens plane in the low optical depth limit $(\tau \ll 1)$. The combination of individual lenses produces several important effects. First, as we discussed previously, the lens distribution as a whole induces shear in the neighborhoods of the individual point masses. For a point mass perturbed by weak shear, we have found in $\S \S 3.1-2$ that the shape of the iso-magnification contours has a universal form - every lens produces a caustic with the same astroid shape (Fig. 2) — and that the normalized differential cross-section $\varphi$ is a 
function of the combination $S A$ only (Fig. 3). Since the magnitude of the shear $S$ varies from lens to lens, there is a distribution of $S$ for the cross-section $\sigma(A \mid S)=\sigma_{0}(A) \varphi(S A)$ near each point mass. In this subsection, we consider the superposition of the cross-sections, with the caustic-induced features at different $A \sim 1 / S$. Other effects of the combination of individual lenses will be considered systematically in subsequent sections.

Under low optical depth condition, the point masses are well separated, and the caustics and the high-magnification contours on the observer plane are relatively isolated. Consequently, the macroimage magnification distribution $P(A)$ at high $A$ can be approximated as a superposition of the cross-sections of the individual point mass plus weak shear lenses:

$$
P(A)=n \int_{0}^{\infty} d S p(S) \sigma(A \mid S)
$$

where $p(S)$ is the probability distribution of the shear due to the other lenses on the plane. For a random lens distribution with optical depth $\tau=\pi n x_{E}^{2}, p(S)$ is given by (Nityananda \& Ostriker 1984; Schneider 1987b; Lee \& Spergel 1990)

$$
p(S)=\frac{\tau S}{\left(\tau^{2}+S^{2}\right)^{3 / 2}}
$$

Note that the shear distribution tends to $p(S)=\tau / S^{2}$ for $S \gg \tau$, which is just the shear from the nearest neighbor, and that $p(S)$ has a prominent peak at $S=\tau / \sqrt{2}$ for $\tau \ll 1$.

Substituting equation (30) into equation (29), we obtain

$$
P(A)=n \sigma_{0}(A) f_{1}(\tau A)=\frac{2 \tau}{\left(A^{2}-1\right)^{3 / 2}} f_{1}(\tau A)
$$

where we have introduced the function

$$
f_{1}(\tau A)=\int_{0}^{\infty} d y \frac{\varphi(\tau A y) y}{\left(1+y^{2}\right)^{3 / 2}}=1+\int_{0}^{\infty} d y \frac{[\varphi(\tau A y)-1] y}{\left(1+y^{2}\right)^{3 / 2}}
$$

to describe the caustic-induced feature in the macroimage magnification distribution. Since $f_{1}(\tau A)$ depends on $\tau$ and $A$ only through the combination $\tau A$, the caustic-induced feature in $P(A)$ also has a simple scaling property. The function $f_{1}(\tau A)$, obtained numerically with $\varphi(S A)$ as shown in Figure 3, is plotted in Figure 4 (solid curve). There is a mild "bump" located at $A \approx 2 / \tau$, which is a $20 \%$ enhancement at its peak. The bump is similar in shape to the features in $\varphi$, but it is much weaker and smoother because of the convolution over the shear distribution. The function $f_{1}(\tau A)$ provides a semi-analytic description for the caustic-induced bump found numerically by Rauch et al. (1992), but only in the low optical depth limit (see $\S 5$ ). 
In Figure 4 we also show an analytic fit (dotted curve) to the numerically evaluated $f_{1}(\tau A)$ :

$$
f_{1}(\tau A)=1-0.81 \frac{(\tau A)^{2}(1-3 \tau A)}{\left[1+1.5(\tau A)^{3 / 2}\right]^{8 / 3}} .
$$

The maximum deviation of this fit from the numerical result is less than 0.01 .

\section{MODIFICATION OF $P(A)$ AT LOW MAGNIFICATION}

The superposition of the cross-sections of individual lenses that results in the causticinduced feature $f_{1}(\tau A)$ is only valid for the magnifications seen by the observers close to one of the astroid-shaped caustics and this, as it turns out, implies the range $A-1 \gg \tau^{2}$. Equation (2) also requires modification at low magnification (i.e., at $\delta A=A-1 \ll 1$ ) as it diverges as

$$
P(A) \approx \frac{\tau}{\sqrt{2}(\delta A)^{3 / 2}}
$$

at $A=1$ and is not normalizable.

For the modification of $P(A)$ at low magnification, we are concerned with the observers who do not lie particularly near an astroid. These observers see one primary image which is barely deflected from the unperturbed position of the source and a large number of faint secondary images, one close to each lens, which are produced by strongly deflected rays (the diffuse component). If we choose a coordinate system such that $\boldsymbol{x}=0$ for the primary ray, then the lens equation near the primary ray is just equation (12) without the $\boldsymbol{d}_{L}$ term, and the magnification of the primary image (which we shall denote by $A_{0}$ ) is obtained by summing the shear perturbations from the point masses around the primary ray: $A_{0}=\left(1-S^{2}\right)^{-1} \approx 1+S^{2}$, where $S=|\boldsymbol{S}|=\left|\sum \boldsymbol{s}_{k}\right|$ and $\boldsymbol{s}_{k}$ is the shear perturbation from the $k$ th point mass in vector notation (see Appendix B). The magnification of the faint image close to the $k$ th point mass is $A_{k}=x_{E}^{4} /\left|\boldsymbol{x}_{k}\right|^{4}=\left|\boldsymbol{s}_{k}\right|^{2}$. Hence the total excess magnification is

$$
\delta A=A_{0}+\sum_{k} A_{k}-1=\left|\sum_{k} \boldsymbol{s}_{k}\right|^{2}+\sum_{k}\left|\boldsymbol{s}_{k}\right|^{2} .
$$

Since the lenses have a Poisson distribution, the calculation of $P(\delta A)$ is well defined. Schneider (1987b) has obtained an expression for $P(\delta A)$ under the assumption that the two terms in equation (35) are statistically independent. While that gives a turn-over at $\delta A \sim \tau^{2}$, the asymptotic behavior at $\delta A \gg \tau^{2}$ fails to match the normalization of equation (2). This is not altogether surprising since for $\delta A \gg \tau^{2}$, both of the sums in equation (35) are dominated by the nearest lens and the two terms are perfectly correlated. 
In Appendix $\mathrm{B}$, we determine $P(\delta A)$ at low magnification, taking into account the correlations between the primary and secondary images. This is rather complicated as $P(\delta A)$ formally reads

$$
P(\delta A)=\int \cdots \int \prod_{k} d^{2} \boldsymbol{s}_{k} p\left(\boldsymbol{s}_{k}\right) \delta\left[(A-1)-\left|\sum_{k} \boldsymbol{s}_{k}\right|^{2}-\sum_{k}\left|\boldsymbol{s}_{k}\right|^{2}\right],
$$

where $p\left(\boldsymbol{s}_{k}\right)$ is the probability distribution of the shear from the $k$ th point mass and $\delta$ is the Dirac delta function. Although equation (36) cannot be reduced to a simple closed form, we show in Appendix $\mathrm{B}$ that it can be reduced to a single integral, which is a function of the combination $(A-1) / \tau^{2}$ (eq.[B14]). Equation (B14) can be written in the form

$$
P(A)=\frac{\tau}{\sqrt{2}(A-1)^{3 / 2}} g_{1}\left[\frac{(A-1)}{\tau^{2}}\right]=\frac{2 \tau}{\left(A^{2}-1\right)^{3 / 2}} g_{1}\left[\frac{(A-1)}{\tau^{2}}\right],
$$

where we have used the fact that $A+1 \approx 2$ for $\delta A \ll 1$. The correction function $g_{1}\left[(A-1) / \tau^{2}\right]$ takes into account the effect of the combination of individual lenses for small magnification. Numerical integration of equation (B14) gives the function $g_{1}$ and the distribution $P(A)$ plotted in Figure 5. An excellent analytic fit to $g_{1}$ is given by

$$
g_{1}(y)=\frac{y e^{-\pi / 4 y}}{\left(\pi / 2^{3 / 2}+y\right)} .
$$

This fit has the same asymptotes (eq.[B15]) as the integral (B14), and it does not differ from the numerical integration results by more than $0.6 \%$.

The distribution (37) has the following properties. It is a normalized distribution: $\int d A P(A)=1$. It has a sharp peak $P(A) \approx 0.16 / \tau^{2}$ at $\delta A \approx 0.84 \tau^{2}$ (see Fig. 5). For $A-1 \gg \tau^{2}$, it has the asymptote

$$
P(A)=\frac{\tau}{\sqrt{2}(A-1)^{3 / 2}},
$$

which matches the low magnification $(\delta A \ll 1)$ asymptote (eq. [34]) of the distribution (2). Since both distributions (31) and (37) give the same result in two overlapping regimes: $A \ll 1 / \tau$ and $A-1 \gg \tau^{2}$, it is easy to construct a macroimage magnification distribution that takes into account the effects of both the caustics and the diffuse component:

$$
P(A)=\frac{2 \tau}{\left(A^{2}-1\right)^{3 / 2}} f_{1}(\tau A) g_{1}\left[(A-1) / \tau^{2}\right] .
$$

In the appropriate limits, this distribution becomes either equation (31) or (37). 


\section{FINAL FORM OF $P(A)$ AND COMPARISON WITH NUMERICAL SIMULATIONS}

\subsection{Renormalization and the Final Form of $P(A)$}

In $\S 3.3$ we assumed that the surface density of the astroid-shaped caustics on the observer plane is the same as the projected surface density of point masses (see eq. 229]). There is, however, a net convergence of the light rays by the overall lens distribution. This increases the density of astroids by a factor $\bar{A}$, where $\bar{A}=(1-\tau)^{-2}$ is the average magnification on the observer plane. Therefore, there should be an additional factor $\bar{A}$ in the normalization of $P(A)$ in equation (31). In the very low optical depth limit $(\tau \ll 1)$, the correction due to this factor $\bar{A} \approx 1+2 \tau$ is small. However, for optical depths of order 0.1 , the correction is not negligible.

As we noted in $\S 1$, Schneider (1987a) derived an analytic result for $P(A)$ in the high magnification limit, which is exact even for finite optical depths $\tau$. The starting point of his analysis is to consider the probability distribution of the shear on the lens plane $p(S)$ (eq. [30]). Since the magnification for a light ray that encounters shear $S$ on the lens plane is $A=\left|1-S^{2}\right|^{-1}$ and each light ray corresponds to an image in Lagrangian space, the distribution $p(S)$ can be transformed into the probability distribution function, $P_{L}(A)$, for the magnification of the individual images in Lagrangian space. For high magnification, $S=1+\delta S$ with $|\delta S|=1 / 2 A$, so

$$
P_{L}(A)=p(S=1)\left|\frac{d \delta S}{d A}\right|=\frac{\tau}{2\left(1+\tau^{2}\right)^{3 / 2}} A^{-2} .
$$

To obtain the probability distribution function for the magnification in Eulerian space $P(A)$, we have to first multiply $P_{L}(A)$ by the factor $\bar{A} / A$, which takes into account the focusing of the light rays by the lenses (Schneider 1987b; Lee \& Spergel 1990), and then double the magnification $(A \rightarrow 2 A)$, which takes into account that the high magnification events are dominated by a pair of very bright microimages that form close to a fold catastrophe. The final result is

$$
P(A)=\frac{2 \tau \bar{A}}{\left(1+\tau^{2}\right)^{3 / 2}} A^{-3}=\frac{2 \tau}{(1-\tau)^{2}\left(1+\tau^{2}\right)^{3 / 2}} A^{-3},
$$

which has the usual $A^{-3}$ form. Although this is an exact analytic result for finite $\tau$, it is limited in applicability to sufficiently high magnification such that the macroimage is dominated by two microimages. As we saw in $\S 3$, for low $\tau$, one actually has to go to fairly high magnification $(A \gg 1 / \tau)$ for this to be a good approximation. The normalization in equation (42) differs from the normalization $2 \tau$ in equation (40) by the factors $\bar{A}$ and $\left(1+\tau^{2}\right)^{-3 / 2}$. For $\tau \lesssim 0.1, \bar{A} \approx 1+2 \tau$ and $\left(1+\tau^{2}\right)^{-3 / 2} \approx 1-3 \tau^{2} / 2$, and the correction to 
the normalization $2 \tau$ is mainly due to the factor $\bar{A}$. Note that we have already derived the factor $\bar{A}$ with the argument given in the previous paragraph.

We can now collect together the three effects of the combination of individual lenses discussed above - the caustic-induced feature from equation (31), the low magnification modification due to the diffuse component (eq. [37]), and the renormalization due to the net convergence of light rays and multiple streaming (eq. 42]) - and find that for $\tau \ll 1$, the final form of the macroimage magnification distribution due to a two-dimensional distribution of point masses is

$$
P(A)=\frac{2 \tau}{(1-\tau)^{2}\left(1+\tau^{2}\right)^{3 / 2}}\left(A^{2}-1\right)^{-3 / 2} f_{1}(\tau A) g_{1}\left[(A-1) / \tau^{2}\right] .
$$

\subsection{Comparison with Numerical Simulations}

Several authors (e.g., Paczyński 1986a; Rauch et al. 1992; Wambsganss 1992) have used numerical simulations to calculate the macroimage magnification distribution, $P(A)$, produced by a two-dimensional distribution of point masses. In particular, distributions with high resolution in $A$ have been obtained from Monte Carlo simulations by Rauch et al. for $\tau=0.1,0.2$, and 0.3 . We now compare the semi-analytic $P(A)$ derived in this paper, equation (43), to these numerical results. As noted, our derivation assumes low optical depth (i.e., $\tau \ll 1)$. It is not immediately obvious whether optical depths in the range $0.1 \leq \tau \leq 0.3$ are sufficiently small and whether we should expect a good agreement between the semi-analytic and numerical results. In fact, as we shall see, $\tau=0.1$ is not sufficiently small. There are differences between the semi-analytic and numerical results that are indicative of finite optical depth effects. Ideally, comparison should also be made for smaller $\tau$, but numerical $P(A)$ with the required accuracy is not available [the computational requirement increases rapidly with decreasing $\tau$ because the caustic-induced feature shifts to higher $A(\propto 1 / \tau)$ while the amplitude of $P(A)$ at large $A(\propto \tau)$ decreases].

In Figure 6 we show the high resolution $P(A)$ obtained by Rauch et al. for $\tau=0.1$ and 0.2 (histograms). The data are those shown in Figure 3 of Rauch et al. (1992), but they are plotted in the form $\left(A^{2}-1\right)^{3 / 2} P(A)$ and the bin sizes at large $A$ are slightly different. For comparison, the solid lines show the full semi-analytic $P(A)$ (eq. 43), and the dotted lines show the semi-analytic $P(A)$ with the caustic-induced feature only (i.e., eq. 43] without the function $\left.g_{1}\right)$. Note that in a plot of $\left(A^{2}-1\right)^{3 / 2} P(A)$, the semi-analytic distributions without the low magnification modification $g_{1}$ or the caustic-induced feature $f_{1}$ are simply horizontal lines of amplitude $2 \tau(1-\tau)^{-2}\left(1+\tau^{2}\right)^{-3 / 2}$ (dashed lines).

At $\log A<0.1$ (or $\delta A<0.3$ ), the semi-analytic and numerical results for $\tau=0.1$ are 
in good agreement, but the results for $\tau=0.2$ are slightly different in shape and amplitude. Since this range of $A$ is dominated by the low magnification modification $g_{1}$, we conclude that the function $g_{1}$ derived in $\S 4$ is valid for $\tau<0.2$. (Recall that the peak in $P(A)$ produced by $g_{1}$ is located at $\delta A \approx 0.84 \tau^{2}$, which is much less than 0.3 for both cases.) The semianalytic caustic-induced feature also provides a reasonably good fit to the numerical results at $\log A>1$ and 1.2 for $\tau=0.1$ and 0.2 , respectively. At intermediate $A$, there are significant differences between the numerical and semi-analytic results. The numerical distribution is lower than the semi-analytic distribution in the neighborhood of the minimum at $A \approx 2.5$, and it is higher than the semi-analytic distribution at smaller and larger $A$. This pattern of deviations is similar to the cross-section shown in Figure 3 and suggests that the function $f_{1}$ derived in $\S 3$ underestimates the strength of the caustic-induced feature at intermediate A. It is, however, important to note that the differences between the semi-analytic and numerical results decrease in strength with decreasing $\tau$ and should be relatively small for

$\tau \lesssim 0.05$. In the next subsection we shall analyze the cause of the differences at intermediate A.

\subsection{Re-examining the Criterion for Low Optical Depth}

In our analysis of the caustic-induced feature in $P(A)$ for low optical depth $(\S 3)$, we assume that all the point masses are well separated. Then the deflection near each point mass is due to that point mass and the shear perturbation from the other lenses, and an astroidshaped caustic is associated with each point mass. However, since the lenses are randomly distributed on the lens plane, some of the lenses have close neighbor(s), separated by less than a few Einstein radius $x_{E}$. In these cases, the caustics are not isolated astroids but more complicated structures produced by the collective effect of two (or more) point masses. If the surface density (or optical depth) of the lens distribution is low enough that the fraction of lenses with close neighbor(s) is small, the contribution to $P(A)$ by these configurations is negligible, and the analysis in $\S 3$ is valid. For larger $\tau$, however, we have to take into account the more complex configurations and can do so by evaluating the macroimage magnification distribution as a series: $P(A)=P_{1}(A)+P_{2}(A)+\cdots$, where $P_{1}(A)$ is the contribution by the point masses perturbed by shear, $P_{2}(A)$ is the contribution by close pairs of point masses, etc. The first term $P_{1}(A)$ is the distribution derived in $\S 3$ (but with a maximum cutoff in the convolution over shear). The second term $P_{2}(A)$ can be evaluated (approximately) as a convolution of the cross-section $\sigma(A \mid d)$ for two point masses separated by distance $d$ with the probability distribution for $d$ if we consider a point mass and its nearest neighbor as a two-point-mass lens and neglect the perturbation from the other lenses. 
To understand the contribution to $P(A)$ from close pairs of point masses, we must first look at some of the properties of lensing by two equal point masses on a single lens plane (see $\S 3$ of Paper II and Schneider \& Weiss 1986 for details). For consistency with the notation in Paper II, we shall express the Lagrangian separation $d$ between the lenses in units of $\sqrt{2} x_{E}$. In the limit $d \gg 2$, the region near each of the point masses is perturbed by the weak shear $\left(S=1 / 2 d^{2}\right)$ from the other point mass, and there are two astroid-shaped caustics. The caustics move towards each other (and become asymmetric) as $d$ decreases, and they touch when $d=2$. As $d$ decreases below 2 , the number of caustics changes from two to one to finally three (see Fig. 3 of Paper II). In Paper II, this sequence of caustic topologies is denoted as topology types $A^{\prime}$ (for $d>2$ ), $B^{\prime}$ (for $1 / \sqrt{2}<d<2$ ), and $C^{\prime}$ (for $d<1 / \sqrt{2}$ ). In all cases, the normalized differential cross-sections $\varphi(A)$ are qualitatively similar to that for the point mass plus weak shear lens, but the caustic-induced features can be significantly stronger (compare Fig. 4 of Paper II to Fig. 3 of this paper). As in the point mass plus weak shear case, there is a discontinuous jump in $\varphi(A)$ at the minimum magnification $A_{\min }$ inside the caustic(s). In the limit $d \gg 2$, the minimum magnification inside the two astroid-shaped caustics is $A_{\min }(d) \approx A_{2}\left(S=1 / 2 d^{2}\right)=2 d^{2}$ (eq. 225]). However, as Witt \& Mao (1995) have shown, $A_{\min }$ is a non-monotonic function of $d$ and has a global minimum of 3 when $d=\sqrt{2}$. If we now consider the convolution of these cross-sections with the probability distribution for $d$, it is clear that the resulting distribution $P_{2}(A)$ should show an enhancement in the caustic-induced feature near $A=3$.

In their analysis of the caustic-induced feature in $P(A)$, Rauch et al. (1992) compared the results from the full scale Monte Carlo simulations to the results from a simpler twopoint-mass model (see their Fig. 6 for the $\tau=0.2$ case). Their two-point-mass calculation is in fact an approximate numerical evaluation of $P_{1}(A)+P_{2}(A)$. They found that the twopoint-mass model is able to reproduce partly the caustic-induced feature at intermediate $A$. In particular, the distribution $P(A)$ from the two-point-mass model shows a dip at $A \approx 3$. Rauch et al. suggested that the slightly lower value $(A \approx 2.5)$ of the dip location found in the full Monte Carlo simulations is due to non-negligible contributions from configurations of three or more point masses.

Is there a simple explanation for the relatively strong contribution to $P(A)$ by the collective effect of two (or more) point masses for $\tau$ as small as 0.1? For a Poisson distribution of point masses, the probability that the nearest neighbor to a point mass is at a distance less than $d$ (again in units of $\sqrt{2} x_{E}$ ) is simply $1-\exp \left(-2 \pi n x_{E}^{2} d^{2}\right)$. If we ignore the deflection due to the other lenses, the point mass and its nearest neighbor is a two-point-mass lens. As we mentioned earlier, a two-point-mass lens produces two astroid-shaped caustics only if $d>d_{A B}$, where $d_{A B}=2$ is the separation at which the caustic topology changes from type $A^{\prime}$ to $B^{\prime}$. Therefore, a simple estimate for the fraction of point masses whose caustics are 
not isolated astroids is

$$
P_{\text {na }}(\tau)=1-\exp \left(-2 \pi n x_{E}^{2} d_{A B}^{2}\right)=1-\exp (-8 \tau)
$$

For the contribution to $P(A)$ from $P_{2}(A)$ (and higher order terms) to be negligible, $P_{\text {na }}(\tau) \ll$ 1 or $\tau \ll 1 / 8$. It is immediately clear that $\tau=0.1$ is not sufficiently small for $P_{2}(A)$ to be negligible. For $\tau=0.1$, we estimate that about half of the point masses produce caustic structures that are more complex than the astroid shape: $P_{\mathrm{na}}=0.55$. This is consistent with the illumination pattern shown in Figure 1 of Rauch et al. (1992). In $\S 5.2$, we concluded from the comparison that the contribution from $P_{2}(A)$ should be reasonably small for $\tau \lesssim 0.05$; this corresponds to $P_{\text {na }} \lesssim 0.33$.

\section{SUMMARY}

In this paper we have attempted to build upon the various approaches developed in previous studies in order to develop a systematic theory of gravitational microlensing by a planar distribution of point masses in the low optical depth limit. In particular, we have derived a practical semi-analytic expression for the probability distribution of macroimage magnification, $P(A)$.

At high magnification $\left(\delta A \gg \tau^{2}\right)$, we model the illumination pattern as a superposition of the patterns due to individual "point mass plus weak shear" lenses. The shear perturbation near each point mass is induced by the neighboring point masses. It breaks the degeneracy of the caustic of an isolated point mass lens and produces an astroid-shaped caustic. The convolution of the magnification cross-section of the point mass plus weak shear lens with the probability distribution of shear yields $P(A)=2 \tau\left(A^{2}-1\right)^{-3 / 2} f_{1}(\tau A)$, where the function $f_{1}(\tau A)$ (eq. 32]; Fig. 4) describes the caustic-induced feature in the macroimage magnification distribution. In effect, $f_{1}(\tau A)$ introduces a mild "bump," a $20 \%$ enhancement, at $A \approx 2 / \tau$. Since $f_{1}(\tau A)$ depends on $\tau$ and $A$ only through the combination $\tau A$, the caustic-induced feature in $P(A)$ exhibits a simple scaling property. To facilitate future computations, we have provided a useful analytic fit to $f_{1}(\tau A)$ (eq. 33. $)$.

We should point out that the results derived in $\S \S 3.1-2$ for the point mass plus weak shear lens may also have applications in the analysis of gravitational microlensing by physical binary systems. Microlensing searches towards the Galactic bulge and the Large Magellanic Cloud have already discovered microlensing events by close binaries with a single merged caustic (Udalski et al. 1994; Alard, Mao, \& Guibert 1995). Since wide binaries are more common than close ones, there should be a significant number of events due to wide binaries

(Di Stefano \& Mao 1996). If the components of the binary are sufficiently far apart, the 
region near each point mass is perturbed by the weak shear from the other point mass. In these cases, there are two separate astroid-shaped caustics, and the results derived in $\S \S 3.1-2$ are applicable.

At low magnification $(\delta A \ll 1)$, the macroimage consists of a bright primary image barely deflected from the unperturbed position of the source and a large number of faint secondary images (the diffuse component) formed close to each of the lenses. The magnifications of the primary and the secondary images can be strongly correlated. Taking into account the correlations, we find that $P(A)=2 \tau\left(A^{2}-1\right)^{-3 / 2} g_{1}\left[(A-1) / \tau^{2}\right]$, where $g_{1}$ (eqs. [37] and [B14]; Fig. 5) represents the low magnification correction. An excellent analytic fit to $g_{1}$ is given by equation (38). The function $g_{1}$ prevents $P(A)$ from diverging at $A=1$, and it introduces a sharp peak of amplitude $P(A) \approx 0.16 / \tau^{2}$ at $A-1 \approx 0.84 \tau^{2}$.

We also discussed the renormalization of $P(A)$ due to the net convergence of light rays and multiple streaming. Finally, collecting together the above results, we find that in the low optical depth limit $(\tau \ll 1)$,

$$
P(A)=\frac{2 \tau}{(1-\tau)^{2}\left(1+\tau^{2}\right)^{3 / 2}}\left(A^{2}-1\right)^{-3 / 2} f_{1}(\tau A) g_{1}\left[(A-1) / \tau^{2}\right] .
$$

In order to determine the realm of validity of the above semi-analytic expression, we compared it against $P(A)$ obtained from Monte Carlo simulations by Rauch et al. (1992) for $\tau=0.1$ and 0.2. At low magnifications $(\log A<0.1)$, we find that the $\tau=0.1$ semi-analytic and numerical results are in good agreement with each other. At greater optical depths, differences arise both in shape and amplitude. We, therefore, conclude that the low magnification modification $g_{1}$ is valid for $\tau<0.2$. The numerical and the semi-analytic results are also in good agreement in the high magnification regime $(\log A>1$ and 1.2 for $\tau=0.1$ and 0.2 , respectively). At intermediate $A$, however, the semi-analytic expression does not match the numerical result even for optical depth $\tau=0.1$. The deviations arise because the function $f_{1}$ derived in $\S 3$ underestimates the strength of the caustic-induced feature at intermediate $A$. The deviations tend to diminish with decreasing optical depth, and we expect them to be relatively small for $\tau \lesssim 0.05$.

In order to understand the discrepancy between the numerical and semi-analytic results for $\tau$ as small as 0.1 , we re-examined our derivation of the function $f_{1}$ describing the causticinduced feature in $P(A)$. In our derivation, we assume that a unique astroid-shaped caustic is associated with each point mass. In a random distribution of point masses, there may arise groups of two (or more) lenses that lie sufficiently close to each other and give rise to caustic configurations more complicated than isolated astroids. If the surface density of point masses is small, such lens configurations will be rare. If, however, the surface density is large, they will be more common and their contributions cannot be neglected. For a distribution of 
optical depth $\tau$, we estimate that the fraction of point masses whose caustics are not simple astroids is $P_{\text {na }}(\tau)=1-\exp (-8 \tau)$. For $\tau=0.1$, about half of the point masses fall into this category. For the purpose of computing the macroimage magnification distribution $P(A)$ in the manner we did, a simple criterion for low optical depth is $P_{\text {na }} \ll 1$ or $\tau \ll 1 / 8$.

In the accompanying Paper II, we extend our analysis to the more complicated situation of a three-dimensional distribution of point mass lenses.

We thank K. Rauch for providing us with the results of Rauch et al. (1992). We also thank S. Mao and K. Rauch for helpful discussions. A.B. and L.K. acknowledge the hospitality shown to them at CITA during their visits in 1995. This work was supported in part by NSERC (Canada), the CIAR cosmology program, CITA, the Institute for Astronomy (L.K.), the Dudley Observatory (A.B.), and a CITA National Fellowship (M.H.L.). 


\section{A. LENS EQUATIONS FOR POINT MASS PLUS ARBITRARY SHEAR}

Expressing $\boldsymbol{r}^{\prime}$ and $\boldsymbol{x}$ in polar coordinates $\left(r^{\prime}, \phi\right)$ and $(x, \vartheta)$ respectively, equation (15) can be written as a fourth order equation for the Lagrangian coordinate $\boldsymbol{x}$ in terms of the Eulerian coordinate $\boldsymbol{r}^{\prime}$ :

$$
\begin{gathered}
(\zeta-1)^{2}\left[\zeta^{2}-\left(2+\varrho^{2}\right) \zeta+1\right]= \\
\tan \vartheta=\left(\frac{1-\zeta-S \zeta}{1-\zeta+S \zeta}\right) \tan \phi
\end{gathered}
$$

where we have defined $\zeta \equiv\left(x / x_{E}\right)^{2}$ and $\varrho \equiv r^{\prime} / x_{E}$. In this notation, the Jacobian of the mapping is

$$
|\widehat{\mathcal{D}}|=\left|\frac{\partial \boldsymbol{r}^{\prime}}{\partial \boldsymbol{x}}\right|=1-\frac{1}{\zeta^{2}}-\frac{2 S \cos 2 \vartheta}{\zeta}-S^{2} .
$$

The critical line (i.e., the loci of Lagrangian points where the Jacobian $|\widehat{\mathcal{D}}|$ vanishes) is given by

$$
\zeta=\frac{S}{1-S^{2}}\left(\cos 2 \vartheta+\sqrt{\cos ^{2} 2 \vartheta+\frac{1-S^{2}}{S^{2}}}\right),
$$

which corresponds to the so-called "Cassini oval." The associated caustic is non-trivial and it is not a point singularity. Based on equations (A1) and (A2), we expect four images in the interior of the caustic and two outside.

\section{B. LOW MAGNIFICATION LIMIT OF $P(A)$}

Let us consider a random distribution of $N=\pi n R^{2}$ point mass lenses within a circle of Lagrangian radius $R$, where $n$ is the projected surface number density of lenses. The total shear at the origin due to the $N$ point masses is given in matrix form by equation (13b). We shall find it convenient to write the two distinct components of the shear matrix $\widehat{\mathcal{S}}_{L}$ as a vector: $\boldsymbol{S}=\sum_{k=1}^{N} \boldsymbol{s}_{k}$, where $\boldsymbol{s}_{k}=\left(x_{E} /\left|\boldsymbol{x}_{k}\right|\right)^{2}\left(\cos 2 \phi_{k}, \sin 2 \phi_{k}\right)$ is the shear perturbation from the $k$ th point mass.

To determine the low magnification limit of $P(A)$, we need to evaluate the probability distribution function for the following combination of shears (eq.[35]):

$$
\delta A=A-1=\left|\sum_{k=1}^{N} \boldsymbol{s}_{k}\right|^{2}+\sum_{k=1}^{N}\left|\boldsymbol{s}_{k}\right|^{2} .
$$

The first term in equation (B1) is just $S^{2}=|\boldsymbol{S}|^{2}$, and the probability distribution of this term alone is found easily from the distribution (30). The second term in equation (B1) is the 
so-called diffuse component, and its probability distribution has previously been considered by Schneider (1987b). The evaluation of the probability distribution of the sum of these two terms, which is $\delta A$, is more complicated.

Let us start with the formal expression for the probability distribution of $\delta A$ as a function of the $N$ statistically independent shears (eq. [36]):

$$
P(\delta A)=\int \cdots \int \prod_{k} d^{2} \boldsymbol{s}_{k} p\left(\boldsymbol{s}_{k}\right) \delta\left[(A-1)-\left|\sum_{k=1}^{N} \boldsymbol{s}_{k}\right|^{2}-\sum_{k=1}^{N}\left|\boldsymbol{s}_{k}\right|^{2}\right],
$$

where $p\left(\boldsymbol{s}_{k}\right)$ is the probability distribution of the shear from the $k$ th point mass and $\delta$ is the Dirac delta function. Equation (B2) can be simplified if we express each term in the integrand as a Fourier transform. For $p\left(\boldsymbol{s}_{k}\right)$, we have

$$
p\left(\boldsymbol{s}_{k}\right)=\frac{1}{(2 \pi)^{2}} \int d^{2} \boldsymbol{t}_{k} q\left(\boldsymbol{t}_{k}\right) e^{-i \boldsymbol{t}_{k} \cdot \boldsymbol{s}_{k}}
$$

where the characteristic function $q\left(\boldsymbol{t}_{k}\right)$ is (Nityananda \& Ostriker 1984)

$$
q\left(\boldsymbol{t}_{k}\right)=1-\frac{\tau}{N} t_{k}
$$

and $\tau=\pi n x_{E}^{2}$ is the optical depth. Because of the cross terms in $|\boldsymbol{S}|^{2}$, it is inconvenient to use the direct Fourier transform of the delta function in equation (B2). Instead, one can introduce an auxiliary integration to express the delta function in the form

$$
\begin{aligned}
\delta & {\left[(A-1)-\left|\sum_{k=1}^{N} \boldsymbol{s}_{k}\right|^{2}-\sum_{k=1}^{N}\left|\boldsymbol{s}_{k}\right|^{2}\right] } \\
& =\int d^{2} \boldsymbol{X} \delta\left[(A-1)-|\boldsymbol{X}|^{2}-\sum_{k=1}^{N}\left|\boldsymbol{s}_{k}\right|^{2}\right] \delta^{2}\left(\boldsymbol{X}-\sum_{k=1}^{N} \boldsymbol{s}_{k}\right) \\
& =\frac{1}{(2 \pi)^{3}} \int d^{2} \boldsymbol{X} \int d v \int d^{2} \boldsymbol{u} \exp \left[-i v\left(w-X^{2}-\sum_{k=1}^{N} s_{k}^{2}\right)-i \boldsymbol{u} \cdot\left(\boldsymbol{X}-\sum_{k=1}^{N} \boldsymbol{s}_{k}\right)\right],
\end{aligned}
$$

where $\delta^{2}$ is the two-dimensional delta function and $w=A-1$. Substituting equations (B3) and (B5) into equation (B2), we can perform the integration with respect to $\boldsymbol{s}_{k}$ :

$$
\int d^{2} \boldsymbol{s}_{k} \exp \left[i v s_{k}^{2}-i\left(\boldsymbol{t}_{k}-\boldsymbol{u}\right) \cdot \boldsymbol{s}_{k}\right]=\frac{i \pi}{v} \exp \left(-i\left|\boldsymbol{t}_{k}-\boldsymbol{u}\right|^{2} / 4 v\right)
$$

and then the integration with respect to $\boldsymbol{t}_{k}$ :

$$
\frac{1}{(2 \pi)^{2}} \int d^{2} \boldsymbol{t}_{k} q\left(\boldsymbol{t}_{k}\right)\left(\frac{i \pi}{v}\right) \exp \left(-i\left|\boldsymbol{t}_{k}-\boldsymbol{u}\right|^{2} / 4 v\right)=1-\frac{f(v, u)}{N},
$$


where

$$
\begin{aligned}
f(v, u) & =\frac{i \tau}{2 v} e^{-i u^{2} / 4 v} \int_{0}^{\infty} d t J_{0}\left(\frac{t u}{2 v}\right) t^{2} e^{-i t^{2} / 4 v} \\
& =\tau \sqrt{\frac{\pi|v|}{2}}\left(1-i \frac{v}{|v|}\right) g(z)
\end{aligned}
$$

and $J_{0}$ is the zero-order Bessel function. In equation (B8), $z=i u^{2} / 8 v$,

$$
g(z)=\left[(1+2 z) I_{0}(z)+2 z I_{1}(z)\right] e^{-z}
$$

and $I_{0}$ and $I_{1}$ are the modified Bessel functions.

We can now group together the $N$ integrations over $\boldsymbol{t}_{k}$ (eq.[B7]) to obtain

$$
\left[1-\frac{f(v, u)}{N}\right]^{N}=e^{-f(v, u)}
$$

in the limit $N \rightarrow \infty$. Then

$$
P(\delta A)=\frac{1}{(2 \pi)^{3}} \int d^{2} \boldsymbol{X} \int d v \int d^{2} \boldsymbol{u} \exp \left[-i v\left(w-X^{2}\right)-i \boldsymbol{u} \cdot \boldsymbol{X}-f(v, u)\right] .
$$

In equation (B11), the integration with respect to $\boldsymbol{X}$ is

$$
\int d^{2} \boldsymbol{X} e^{i\left(v X^{2}-\boldsymbol{u} \cdot \boldsymbol{X}\right)}=\frac{i \pi}{v} e^{-i u^{2} / 4 v},
$$

and we are left with two integrations with respect to $v$ and $u$. Substituting equations (B8) and (B12) into equation (B11), and using variables $v$ and $z$ instead of $v$ and $u$, we obtain

$$
P(\delta A)=\frac{2}{\pi} \int_{0}^{\infty} d z e^{-2 z} \int_{0}^{\infty} d v \cos \left[w v-\tau \sqrt{\frac{\pi v}{2}} g(z)\right] \exp \left[-\tau \sqrt{\frac{\pi v}{2}} g(z)\right] .
$$

Finally, after performing the integration with respect to $v$, we have $P(\delta A)$ in the form

$$
P(\delta A)=\frac{\tau}{w^{3 / 2}} \int_{0}^{\infty} d z g(z) \exp \left[-2 z-\frac{\pi \tau^{2}}{4 w} g^{2}(z)\right]
$$

where $w=\delta A$ and $g(z)$ is defined in equation (B9). Equation (B14) must be integrated numerically in general, but it has the following analytic asymptotes:

$$
\begin{aligned}
P(\delta A) & =\frac{2}{\pi \tau_{1}^{2}} y^{-1 / 2} e^{-\pi / 4 y} & & \text { for } y=\delta A / \tau^{2} \ll 1 \\
& =\frac{1}{\sqrt{2} \tau^{2}} y^{-3 / 2} & & \text { for } y=\delta A / \tau^{2} \gg 1
\end{aligned}
$$




\section{REFERENCES}

Alard, C., Mao, S., \& Guibert, J. 1995, A\&A, 300, L17

Alcock, C., et al. 1993, Nature, 365, 621

Aubourg, E., et al. 1993, Nature, 365, 623

Chang, K., \& Refsdal, S. 1984. A\&A, 132, 168

Di Stefano, R., \& Mao, S. 1996, ApJ, 457, 93

Gurbatov, S. N., Malakhov, A. N., \& Saichev, A. I. 1991, Nonlinear Random Waves and Turbulence in Nondispersive Media: Waves, Rays, Particles (Manchester: Manchester Univ. Press)

Kaiser, N. 1992, in New Insights into the Universe, ed. V. J. Martínez, M. Portilla, \& D. Sáez (Berlin: Springer-Verlag), 247

Kofman, L., Bertschinger, E., Gelb, J. M., Nusser, A., \& Dekel, A. 1994, ApJ, 420, 44

Lee, M. H., Babul, A., Kofman, L., \& Kaiser, N. 1996, ApJ, submitted (Paper II)

Lee, M. H., \& Spergel, D. N. 1990, ApJ, 357, 23

Longuet-Higgins, M. S. 1960, Proc. Camb. Phil. Soc., 56, 27

Mao, S. 1992, ApJ, 389, 63

Nityananda, R., \& Ostriker, J. P. 1984, J. Ap. Astr., 5, 235

Paczyński, B. 1986a, ApJ, 301, 503

—. 1986b, ApJ, 304, 1

—. 1994, Acta Astr., 44, 235

Press, W. H., \& Gunn, J. E. 1973, ApJ, 185, 397

Rauch, K. P., Mao, S., Wambsganss, J., \& Paczyński, B. 1992, ApJ, 386, 30

Schneider, P. 1987a, ApJ, 319, 9

- 1987b, A\&A, 179, 80

Schneider, P., Ehlers, J., \& Falco, E. E. 1992, Gravitational Lenses (New York: SpringerVerlag)

Schneider, P., \& Weiss, A. 1986, A\&A, 164, 237

Turner, E. L., Ostriker, J. P., \& Gott, J. R. 1984, ApJ, 284, 1

Udalski, A., Szymański, M., Mao, S., Di Stefano, R., Kałużny, J., Kubiak, M., Mateo, M., \& Krzemiński, W. 1994, ApJ, 436, L103 
Wambsganss, J. 1992. ApJ, 386, 19

Witt, H. J., \& Mao, S. 1995, ApJ, 447, L105

Young, P. 1981, ApJ, 244, 756

Zel'dovich, Ya. B. 1970, A\&A, 5, 84 
Fig. 1.- A schematic diagram illustrating our notation for single plane gravitational lensing. A light ray that leaves the source with angle $\boldsymbol{\theta}$ has Lagrangian coordinates $\boldsymbol{x}$. It is deflected on the lens plane and reaches the observer plane at Eulerian coordinates $\boldsymbol{r}$.

Fig. 2.- The caustic and the iso-magnification contours on the observer plane for the point mass plus weak shear lens. They were obtained numerically for shear $S=0.02$. The axes are in units of $x_{E} S$. The caustic has the shape of an astroid with the four cusps at a distance $2 x_{E} S$ from the origin. The contours of critical values $A_{1}=2 / 3 \sqrt{3} S$ and $A_{2}=1 / S$ are also indicated.

Fig. 3.- The "normalized" differential cross-section, $\varphi(S A)=\sigma(A \mid S) / \sigma_{0}(A)$, of the point mass plus weak shear lens (solid line). It was obtained numerically for shear $S=0.02$. The dotted lines show the analytic fit, equation (28).

Fig. 4.- The function $f_{1}(\tau A)$ (eq. 32]) that describes the caustic-induced feature in the macroimage magnification distribution, $P(A)$, at low optical depth $\tau$. The solid line is the numerical result using $\varphi(S A)$ shown in Fig. 3, and the dotted line is the analytic fit, equation (33).

Fig. 5.- (a) The function $g_{1}\left[y=(A-1) / \tau^{2}\right]$ (eq. [37]) that describes the modification of $P(A)$ at low magnification in the low optical depth limit. (b) The macroimage magnification distribution $P(A)\left(=g_{1}[y] / \sqrt{2} y^{3 / 2} \tau^{2}\right)$ at low magnification in the low optical depth limit.

Fig. 6.- Comparison of the semi-analytic macroimage magnification distribution, $P(A)$, with the Monte Carlo results of Rauch et al. (1992) for optical depth $\tau=0.1$ and 0.2 (histograms). The solid lines are the semi-analytic $P(A)$ with the caustic-induced feature and the modification at low $A$ (eq. 443), and the dotted lines are the semi-analytic $P(A)$ with the caustic-induced feature only (i.e., eq. 43 without the function $g_{1}$ ). 


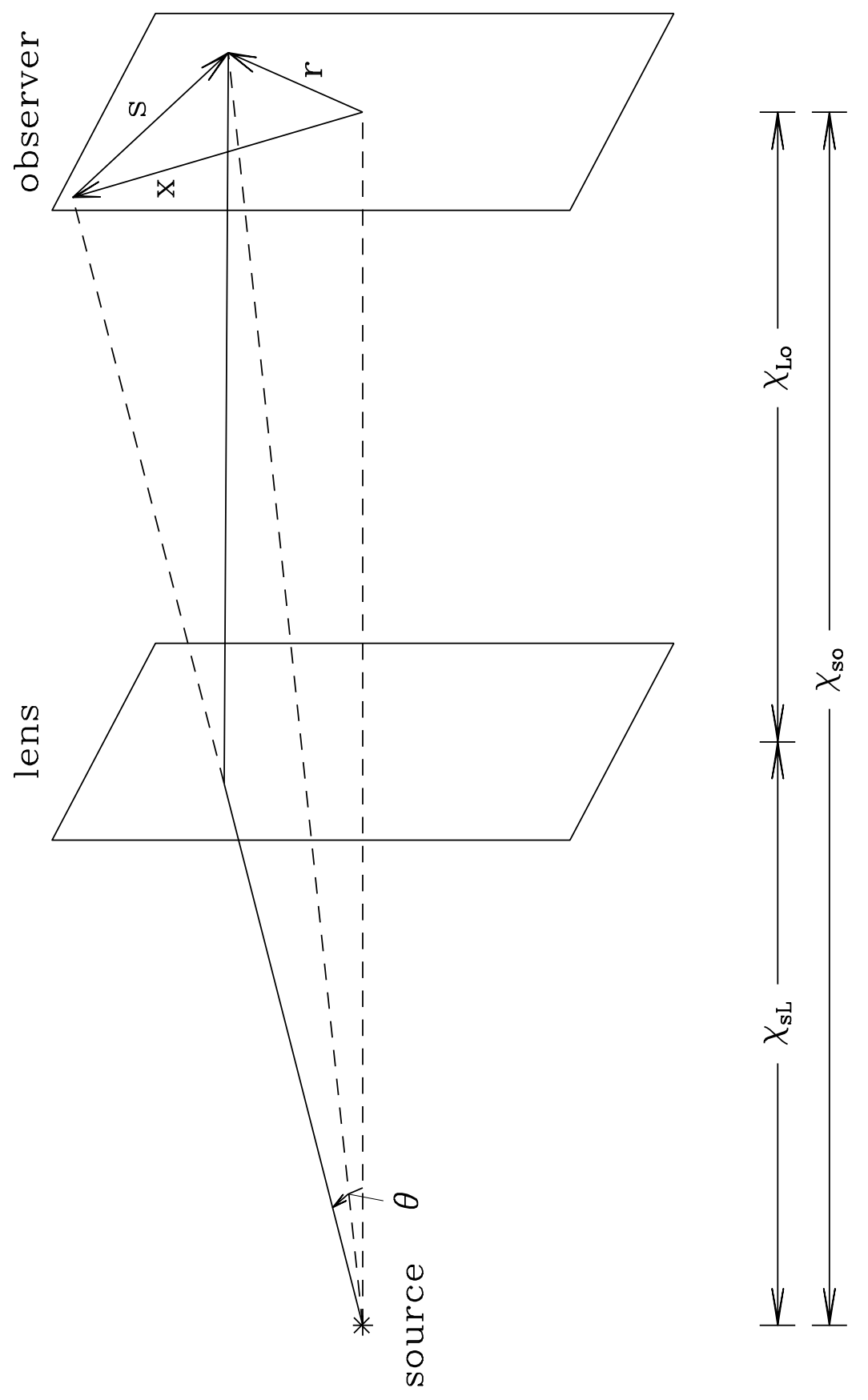




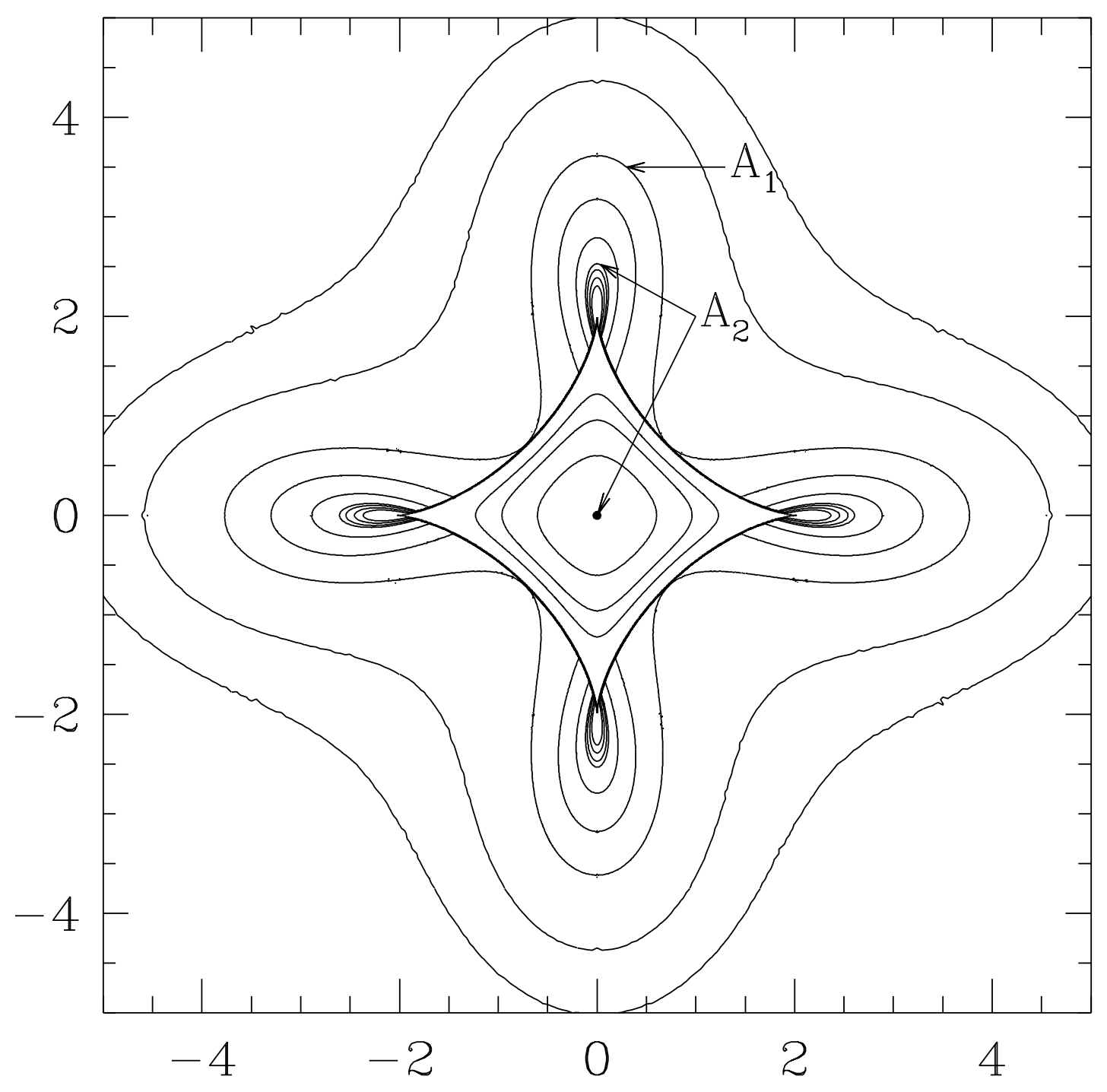




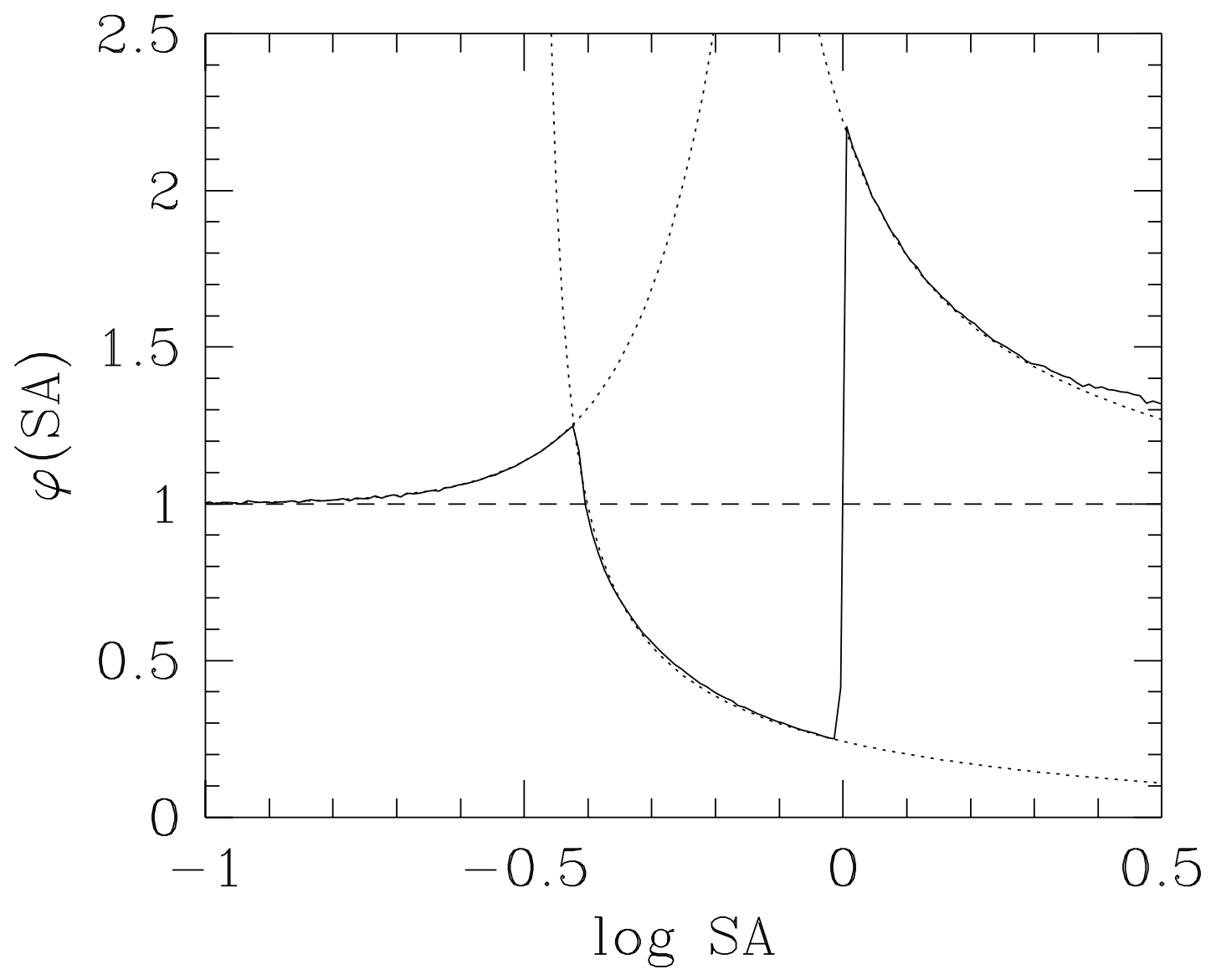




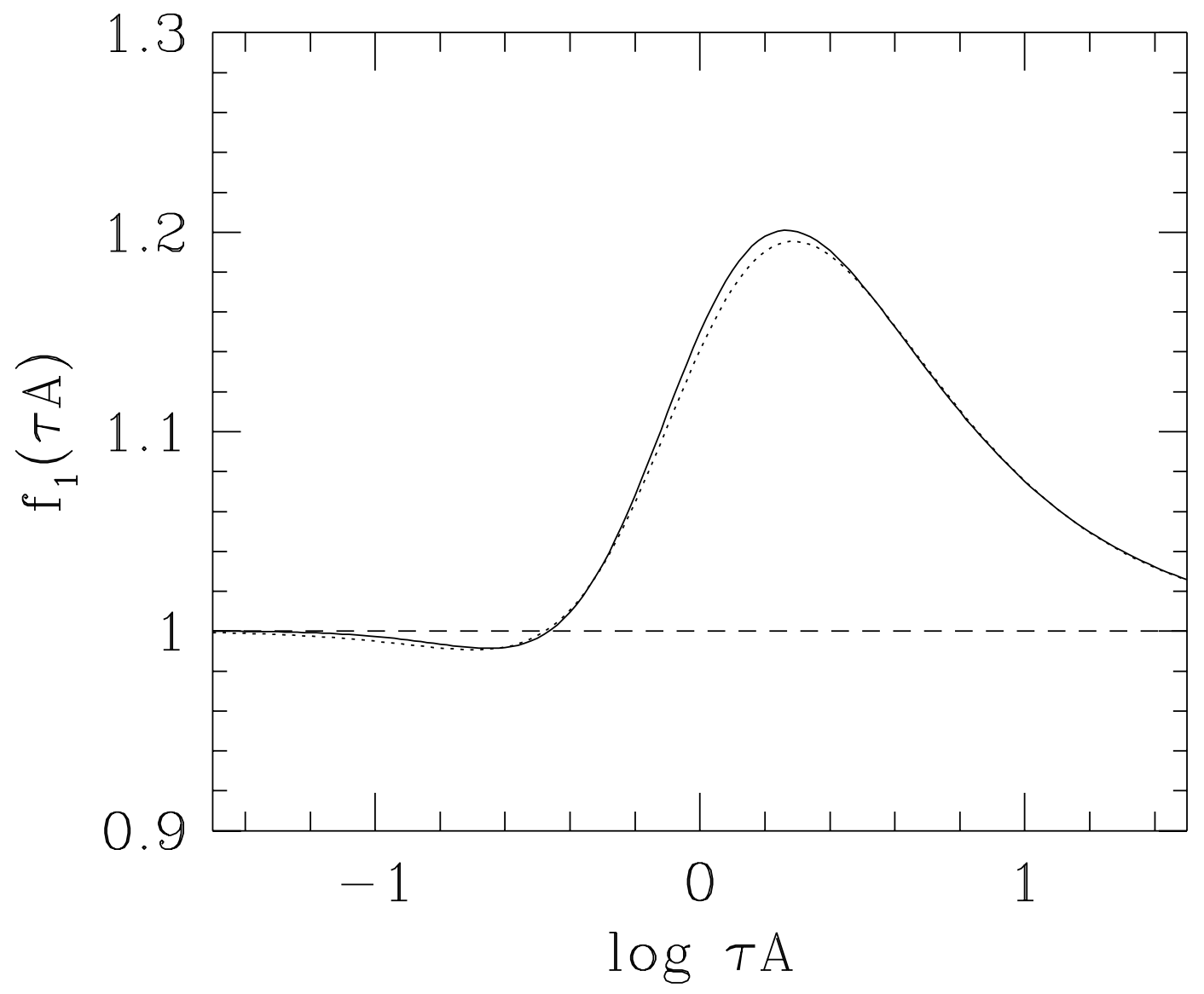




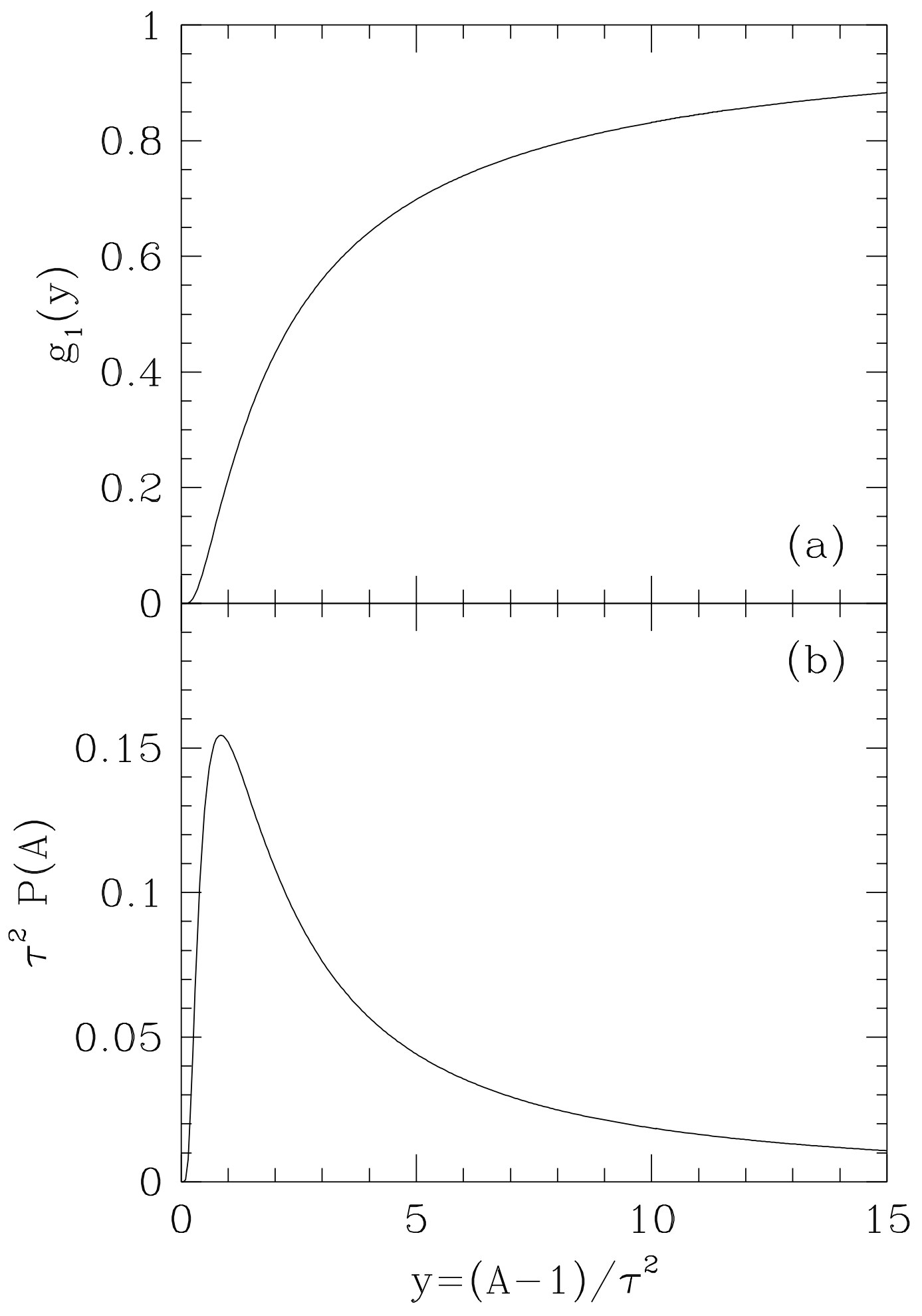




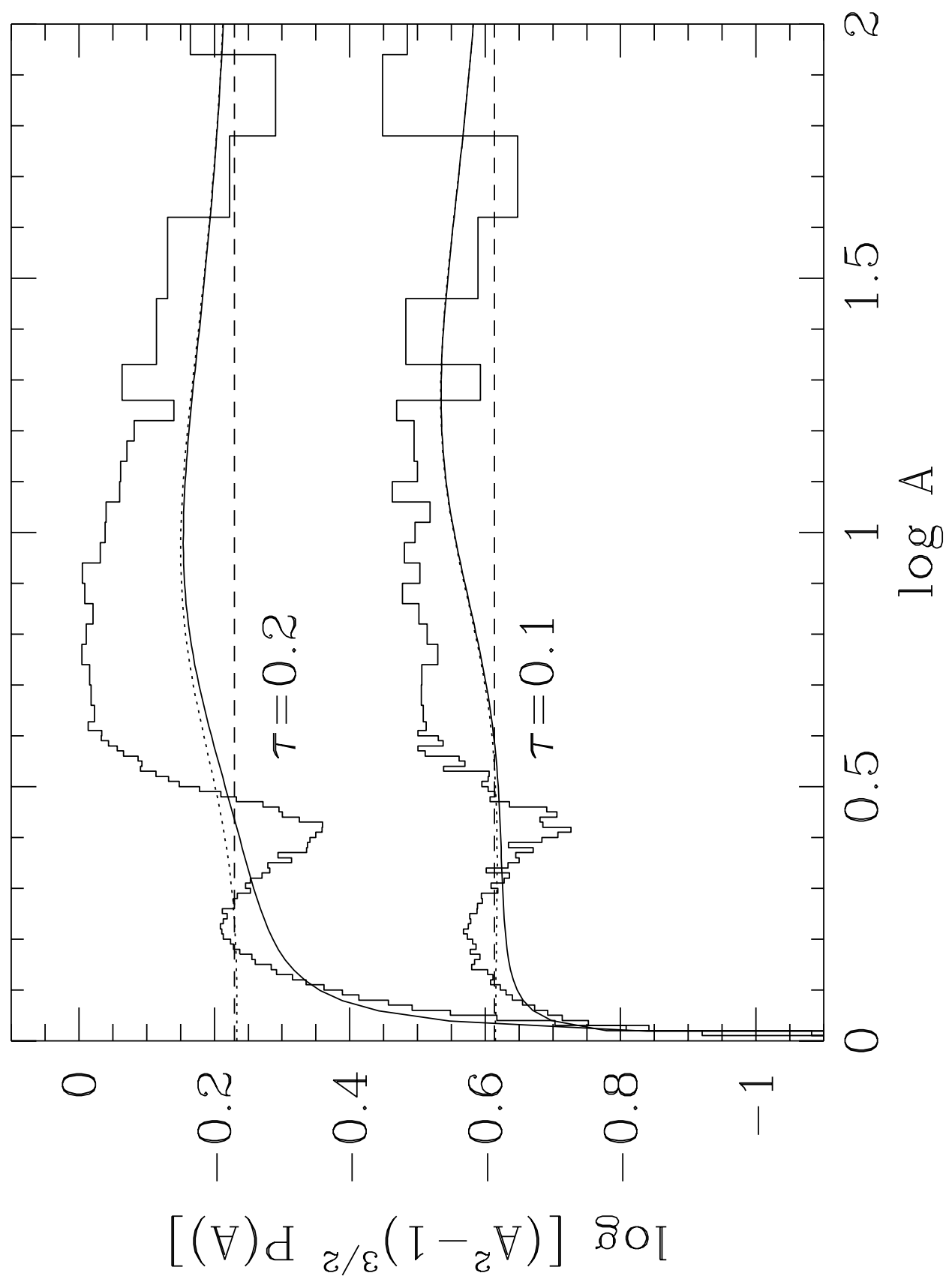

